SNS-PH/00-17

hep-ph/0011385

\title{
Leptogenesis in a Realistic Supersymmetric Model of Inflation with a Low Reheat Temperature
}

\author{
M. Bastero-Gil† and S. F. King* \\ † Scuola Normale Superiore, Piazza dei Cavalieri 7, \\ 56126 Pisa, Italy \\ *Department of Physics and Astronomy, University of Southampton, \\ Southampton, SO17 1BJ, U.K.
}

\begin{abstract}
We discuss leptogenesis in a realistic supersymmetric model of inflation with a low reheat temperature 1-10 GeV. The lepton asymmetry is generated by a decaying right handed sneutrino, which is produced after inflation during preheating. The inflationary model is based on a simple variant of the Next-to-Minimal Supersymmetric Standard model (NMSSM) which solves the $\mu$ problem, called $\phi$ NMSSM, where the additional singlet $\phi$ plays the role of the inflaton in hybrid (or inverted hybrid) type models. The model is invariant under an approximate Peccei-Quinn symmetry which also solves the strong CP problem, and leads to an invisible axion with interesting cosmological consequences. We show how the baryon number of the universe and the nature of cold dark matter are determined by the same parameters controlling the strong CP problem, the $\mu$ problem and the neutrino masses and mixing angles.
\end{abstract}

November 3, 2018 


\section{Introduction}

Leptogenesis is an attractive mechanism which has been proposed to generate the observed baryon asymmetry of the Universe (BAU) [1, 2]. The mechanism involves the out-of-equilibrium decay円 of a heavy right handed neutrino $N_{R}$ (or sneutrino $\tilde{N}_{R}$ [3, 团). The net lepton number L produced in the decay is then reprocessed into baryon number $\mathrm{B}$ by anomalous $(\mathrm{B}+\mathrm{L})$ violating sphaleron interactions, which otherwise conserve (B-L) [6]. The same physics that allows the right handed neutrinos to decay into light leptons is also responsible for a see-saw neutrino mass matrix. Combining the see-saw mechanism with the latest experimental data on neutrino masses [0] seems to favour a scale for the right handed neutrino mass $M_{R}$ in the range $10^{7}-10^{14} \mathrm{GeV}$.

What mainly distinguishes the different scenarios of leptogenesis that can be found in the literature is the production mechanism for the heavy $N_{R}\left(\right.$ or $\left.\tilde{N}_{R}\right)$. The lepton (baryon) asymmetry has to be produced at some early stage in the cosmological evolution of the Universe, at some point after inflation ends and before the time of nucleosynthesis. In a sense, leptogenesis/baryogenesis is closely related to the inflationary dynamics and the post-inflationary reheating era. The right handed sneutrino could be itself the inflaton [3], with L generated during the reheating period. If this is not the case, and the reheating temperature $\left(T_{R H}\right)$ after inflation is larger than $M_{R}$, the heavy (s)neutrinos can be thermally produced after reheating, and the final lepton asymmetry will depend on the out-of-equilibrium conditions at the time they decay [1, 2, 8]. However, in supersymmetric models such a large $T_{R H}$ may be in conflict with the standard bound $T_{R H}<10^{9} \mathrm{GeV}$ in order to avoid an overabundance of gravitinos [9]. On the other hand, if $T_{R H}<M_{R}$ the right handed (s)neutrinos would have to be produced by the out-of-equilibrium inflaton decay, either in perturbative decays [12] or by parametric resonance [13] during preheating. In this way, the outof-equilibrium condition for baryogenesis/leptogenesis is automatically satisfied. The other two requirements, baryon number violation and $\mathrm{C}$ and $\mathrm{CP}$ violation, will be provided by the sphaleron interactions and complex phases in the neutrino Yukawa couplings respectively.

In this paper we shall extend the model for inflation proposed in Ref. [14 in order to include neutrino masses and implement leptogenesis. The inflation model is based on the next-to-minimal supersymmetric model and provides an intermediate scale solution to the $\mu$ problem, and the strong CP problem via the Peccei-Quinn mechanism [14]. The supergravity version of the model [15] solves the $\eta$ problem via the implementation of a no-scale mechanism, provides F-term inflation from the moduli

\footnotetext{
${ }^{1}$ Models of leptogenesis based instead in the cosmological evolution of flat directions which carry lepton number can be found for example in Ref. [4, 5].

${ }^{2}$ Strictly speaking, the condition $T_{R H}<M_{R}$ does not rule out thermal production of heavy neutrinos. In most of inflationary models reheating is not instantaneous, and the maximum temperature $T^{\text {max }}$ reached is usually much larger than $T_{R H}$ 10, and we could have $T_{R H}<M_{R}<T^{\max }$. Like in GUT baryogenesis, there could be models where the decay of the inflaton into heavy neutrinos may be suppressed or forbidden, but still they could be thermally produced during the long period of reheating [11].
} 
fields which are stabilised before and after inflation and there is no moduli problem or gravitino problem. The inflationary model is of the hybrid type, characterised by a not too large scale for the vacuum energy, $V(0)^{1 / 4} \simeq 10^{8} \mathrm{GeV}$ and a very low reheating temperature $O(1 \mathrm{GeV})$. It is interesting to study leptogenesis within models with such a low reheat temperature since in such models thermal production of Majorana neutrinos during or after reheating is impossible, so the production mechanism will rely on preheating the fields which occurs during the oscillatory period following the end of inflation. This model is particularly interesting since the oscillating inflaton fields at the end of inflation do not couple directly to the sneutrinos, but only indirectly via a coupling to the Higgs doublets. Thus the production of sneutrinos during preheating is linked also to the production of Higgs scalars, and since the Higgs scalars decay into both radiation and neutralinos it becomes possible to relate the relic density of the lightest neutralinos to the baryon number of the universe. Relativistic axions are also produced during reheating but these are red-shifted away, although later on non-relativistic axions are additionally produced by the usual misalignment mechanism and these will contribute to cold dark matter.

The main advantage of studying a realistic supersymmetric particle physics model of inflation is that questions such as the nature of cold dark matter and baryogenesis via leptogenesis are related, and determined by the same parameters which control the particle physics questions of the $\mu$ problem, the strong CP problem, and neutrino masses although as we shall see there are many uncertainties at present and many of our estimates will have errors of one or two orders of magnitude.

The layout of the rest of the paper is as follows. In section 2 we summarise the main properties of the model, and introduce the right handed neutrinos with the usual superpotential suitable for neutrino physics and leptogenesis. An estimation of the lepton asymmetry produced is given in section 3. Because the right handed neutrinos will decay in less than a Hubble time, much before the inflaton has time to decay, we need to check how much of the asymmetry survive the reheating era. This is done in section 4 . In section 5 we present our conclusions.

\section{A model for inflation and leptogenesis.}

The model of inflation we have proposed is based on the superpotential [14]:

$$
W=\lambda N H_{1} H_{2}+\kappa \phi N^{2},
$$

where $H_{1}, H_{2}$ are the Higgs doublets and $\phi, N$ are gauge singlets. The superpotential is invariant under a $U(1)_{P Q}$ Peccei-Quinn symmetry, which is broken during and after inflation by the vevs of $\phi$ and $N$. The vacuum energy $V(0)$, needed during inflation, originates from an F-term of the effective sugra theory [15]. The Higgs doublets play no role during inflation, $\phi$ is the inflaton, and $N$ is the second singlet needed to end hybrid inflation. Imposing the slow-rolling and COBE constraints for inflation gives 
the order of magnitude results:

$$
\kappa \sim 10^{-10},\langle\phi\rangle \sim\langle N\rangle \sim 10^{13} \mathrm{GeV}, V(0)^{1 / 4} \sim 10^{8} \mathrm{GeV} H(0) \sim O(\mathrm{MeV}),
$$

with $H(0)$ the Hubble parameter during inflation. In order to have an effective $\mu$ term in the Higgs sector of the correct order of magnitude, we require $\lambda \sim \kappa$. The smallness of the couplings is accounted for by the use of higher dimensional operators, so the superpotential in Eq. 1] should be viewed as an effective superpotential which originates from some intermediate scale solution to the $\mu$ problem, as discussed elsewhere [14]. Due to the smallness of the (effective) couplings, the fields $\phi$ and $N$ are very longlived, decaying mainly into axions with a decay rate $\Gamma_{\phi} \sim 10^{-17} \mathrm{GeV}$. Considering only the standard perturbative reheating period following inflation, this will give rise to a reheating temperature of order a few $\mathrm{GeVs}$, much below the electroweak scale.

Right-handed majorana neutrinos are introduced in the model with the usual superpotential:

$$
W=M_{R_{i}} N_{R_{i}} N_{R_{i}}+\lambda_{L R_{i j}} L_{i} H_{2} N_{R_{j}},
$$

written in the eigenstate basis for the $N_{R_{i}}$. The right handed neutrino masses and Yukawa couplings $\lambda_{L R}$ has to be such that they reproduced the observed properties of the light neutrino spectrum. The recent data from Super-Kamiokande supports $\nu_{\mu} \leftrightarrow \nu_{\tau}$ oscillations with $\sin ^{2} 2 \theta_{23}>0.88$ and a mass squared splitting $\Delta m_{23}^{2} \simeq$ $(1.5-5) \times 10^{-3} \mathrm{eV}^{2}$. It also favours the large mixing angle solution for solar neutrino mixing, with $\sin ^{2} 2 \theta_{12} \sim 0.75$ and $\Delta m_{12}^{2} \sim 2.5 \times 10^{-5} \mathrm{eV}^{2}$, although others solutions are not excluded. For numerical estimations, and as a working example, we will use the results of a recent estimate of all quark and lepton masses and mixing angles based on a string-inspired Pati-Salam model [16], although we shall only be concerned with the leptonic part of this model. Due to the gauged $S U(2)_{R}$ symmetry the model predicts three right-handed neutrinos, and the heaviest one is the one associated with the third family with a mass of $10^{14} \mathrm{GeV}$. Although this is the heaviest it nevertheless plays the dominant role in generating the atmospheric masses and mixing angle, due to the Yukawa structure of the model, leading to an automatic neutrino mass hierarchy, according to the single right handed neutrino dominance mechanism [17], and bi-maximal mixing. In terms of the heaviest right handed scale of the order $M_{R_{3}} \simeq 10^{14} \mathrm{GeV}$, and the Wolfenstein expansion parameter $\lambda \simeq 0.22$, the right handed neutrino masses and couplings are given by:

$$
\begin{aligned}
M_{R_{i}} & \sim 10^{14}\left(\lambda^{9}, \lambda^{5}, 1\right) \mathrm{GeV} \sim\left(10^{8}, 10^{10}, 10^{14}\right) \mathrm{GeV} \\
\lambda_{L R} & \sim\left(\begin{array}{ccc}
\lambda^{8} & \lambda^{4} & \lambda^{2} \\
\lambda^{7} & \lambda^{4} & \lambda \\
\lambda^{7} & \lambda^{4} & 1
\end{array}\right),
\end{aligned}
$$

Note that the lightest right-handed neutrino of mass $10^{8} \mathrm{GeV}$ is significantly lighter than $10^{14} \mathrm{GeV}$ but does not give the dominant contribution to physical neutrino masses due to its suppressed Yukawa couplings, although it is light enough to be produced through preheating. 
Given that in the inflationary model $T_{R H} \ll V(0)^{1 / 4}<M_{R_{i}}$, the $N_{R_{i}}\left(\tilde{N}_{R_{i}}\right)$ fields cannot be produced thermally at any stage, nor in the perturbative decay of $\phi$ and $N$ fields. Therefore, we depend upon preheating for that.

Due to the lack in the superpotential of a direct Yukawa coupling between the singlets superfields $\phi, N$ and $N_{R}$, preheating of the right handed neutrinos does not look possible. The situation is different for the scalar components, because of the coupling $\lambda_{L R}$ with the doublet Higgs $H_{2}$ and the leptons $L_{i}$. This will induce a term in the scalar potential of the form:

$$
V=\cdots+\left|\lambda_{L R}\right|_{k j}^{2}\left|H_{2}\right|^{2} \tilde{N}_{R_{j}} \tilde{N}_{R_{k}}^{*}+\cdots .
$$

Large oscillations in the Higgs fields will be induced through their coupling to the singlets in Eq.1], and in turn we expect this to trigger the preheating of the sneutrinos. The lepton asymmetry will be generated by the decay of the sneutrinos instead of that of the neutrinos. We notice also that the maximum possible $T$ that we can reach during reheating is going to be smaller than the $M_{R_{i}}$ masses (at most $T^{\text {max }} \sim$ $10^{8} \mathrm{GeV} \sim M_{R_{1}}$ ), so once produced, the sneutrinos will remain out of equilibrium and will decay faster than say the inflaton.

\section{Lepton/Baryon asymmetry}

Preheating of right-handed sneutrinos provides the seed we need for leptogenesis, i.e., a non-zero number density of the order

$$
n_{\tilde{N}_{R_{i}}} \sim c_{i} \frac{V(0)}{M_{R_{i}}},
$$

where $c_{i}$ parametrize the fraction of the total vacuum energy which is transfered to the sneutrinos during preheating. Given the hierarchy in masses, it is not unreasonable to assume $c_{3} \ll c_{2}, c_{1}$, whilst we will take $c_{2} \sim c_{1} \sim c \sim O(1-0.1)$. CP violation

in the decay of $\tilde{N}_{R_{i}}$ comes from the interference between the tree-level and one-loop amplitudes [2, 8, 18, 19]. The $\mathrm{CP}$ asymmetries given by the interference with the one-loop vertex amplitude are [2, 国:

$$
\begin{aligned}
\epsilon_{i} & =\frac{\Gamma\left(\tilde{N}_{R_{i}} \rightarrow \tilde{l}+H_{2}\right)-\Gamma\left(\tilde{N}_{R_{i}}^{\dagger} \rightarrow \tilde{l}^{\dagger}+H_{2}^{\dagger}\right)}{\Gamma\left(\tilde{N}_{R_{i}} \rightarrow \tilde{l}+H_{2}\right)+\Gamma\left(\tilde{N}_{R_{i}}^{\dagger} \rightarrow \tilde{l}^{\dagger}+H_{2}^{\dagger}\right)} \\
& =\frac{1}{8 \pi\left(\lambda_{L R}^{\dagger} \lambda_{L R}\right)_{11}} \sum_{j}\left(\operatorname{Im}\left[\left(\lambda_{L R}^{\dagger} \lambda_{L R}\right)_{1 j}\right]^{2}\right) f\left(M_{R_{j}}^{2} / M_{R_{i}}^{2}\right),
\end{aligned}
$$

where

$$
f(x)=\sqrt{x}\left[1-(1+x) \log \left(\frac{1+x}{x}\right)\right] .
$$

The interference with the absorptive part of the one-loop self-energy also gives a contribution to the asymmetry, which in general is the same order as those given 
above, unless the (s)neutrinos were almost degenerate in which case could it be much larger [18, 19].

As an example in order to estimate the values of $\epsilon_{i}$, we will consider the model given in Eqs. 4 and 5. Assuming maximal CP violation $\left(\operatorname{Im}[\cdots]^{2} \sim|\cdots|^{2}\right)$, we can see that the asymmetries will be dominated by the larger couplings to the third generation of leptons, with:

$$
\begin{aligned}
\epsilon_{1} & \sim \frac{\left|\lambda_{L R}\right|_{33}^{2}}{8 \pi} \frac{M_{R_{1}}}{M_{R_{3}}} \sim \frac{\lambda^{9}}{8 \pi} \sim 10^{-7}-10^{-8}, \\
\epsilon_{2} & \sim \frac{\left|\lambda_{L R}\right|_{33}^{2}}{8 \pi} \frac{M_{R_{2}}}{M_{R_{3}}} \sim \frac{\lambda^{4}}{8 \pi} \sim 10^{-4}-10^{-5}, \\
\epsilon_{3} & \sim \frac{\left|\lambda_{L R}\right|_{32}^{2}}{8 \pi} \frac{M_{R_{2}}}{M_{R_{3}}} \sim \frac{\lambda^{13}}{8 \pi} \sim 10^{-10}-10^{-11} .
\end{aligned}
$$

We remark again that these are only order of magnitudes estimations, with large uncertainties in their values. Besides, the values of $\epsilon_{i}$ are model dependent. Other texture models with values of the Yukawas conistent with the experimental data on neutrinos, and similar hierarchy among the right handed neutrino masses, could give rise to a larger asymmetry such as $\epsilon_{1} \sim 10^{-6}[20]$.

The decay of the sneutrinos occurs fast enough to neglect any effect due to the expansion of the Universe, and the lepton asymmetry is then given by:

$$
n_{B-L} \simeq \epsilon_{i} n_{\tilde{N}_{R_{i}}} \approx \epsilon_{i} c_{i} \frac{V(0)}{M_{R_{i}}} .
$$

The decay of the heaviest right handed sneutrino (if produced) will give rise to a negligible lepton asymmetry, whilst that generated in the decay of $\tilde{N}_{R_{1}}$ and $\tilde{N}_{R_{2}}$ are comparable because $\epsilon_{1} / M_{R_{1}} \sim \epsilon_{2} / M_{R_{2}} \sim 1 / M_{R_{3}}$. This is then converted into baryon number by $\mathrm{B}+\mathrm{L}$ violating sphalerons interactions (which are in equilibrium for temperatures in the interval $\sim\left[200,10^{12}\right] \mathrm{GeV}$ ] [21],

$$
n_{B}=-\frac{8}{23} n_{B-L}
$$

and finally at the time of nucleosynthesis we will have:

$$
\left.\frac{n_{B}}{s}\right|_{\text {nucl. }} \approx \frac{8}{23} \gamma \epsilon_{i} \frac{(c V(0))^{1 / 4}}{M_{R_{i}}} \sim \frac{\gamma}{23 \pi}\left|\lambda_{L R}\right|_{33}^{2} \frac{(c V(0))^{1 / 4}}{M_{R_{3}}}
$$

where we have used $s=\left(2 \pi^{2} / 45\right) g_{*} T^{3}$ evaluated at the time the leptons are produced, i.e., $T \simeq .3(\mathrm{cV}(0))^{1 / 4}$, with the effective number of relativistic degrees of freedom $g_{*} \sim 100$. The factor $\gamma$ accounts for the dilution due to possible entropy production during reheating. Substituting the values of $V(0)$ and $M_{R_{3}}$ Eqs. (2) and (4), with $\lambda_{L R_{33}} \simeq 1$, we obtain:

$$
\left.\frac{n_{B}}{s}\right|_{n u c l .} \sim c^{1 / 4} \times 10^{-8} \gamma
$$

In order to explain the observed baryon asymmetry $n_{B} / s \sim 10^{-10}$ [22] we cannot allowed much entropy (radiation) production during the reheating era. In the next, we will try to estimate the factor $\gamma$ based on simple assumptions. 


\section{Preheating/Reheating}

During the oscillations of the background fields $\phi$ and $N$, particles can be produced by parametric resonance (preheating) [23, 24, 25] much before the inflaton has time to decay perturbatively, being this in general a more efficient mechanism of particle production than standard perturbative decay.

Even if the couplings are very small, the amplitude of the oscillations is large enough to "preheat" the modes of the scalar fields $\phi$ and $N$. Beside, the value of Hubble parameter is small in the model, which allows for a large number of oscillations in a Hubble time, before they start to feel the effect of the expansion. Because hybrid inflation ends in a phase transition, with the effective squared mass of the $N$ field changing sign, production of $\phi$ and $N$ quanta is very efficient during the first few oscillations of the background fields 26]. Due to the coupling $\lambda$ between the Higgses and the $N$ field, we also expect to preheat the Higgs fields $H_{i}$ in a similar way. The evolution equations for the Higgs quantum fluctuations are indeed analogous to those of the singlets, and we can assume similar number densities for both. The sneutrino fields $\tilde{N}_{R_{i}}$ are therefore preheated through the Higgses, with the lightest one more likely to be produced, and they will be clearly out of equilibrium. We may also preheat axions, fermions, etc.. but with much smaller number densities?.

Preheating is efficient only in producing very low frequency modes. Nevertheless, rescattering effects will allow to excite higher frequency modes and redistribute the energy density. Based on the results for the singlets [26], we may estimate that after just 3-4 oscillations a fraction of the vacuum energy has been transfered to the singlets and the other fields quanta, with more or less equal energy densities. The typical time scale for this to happen is given by:

$$
\Delta t_{\text {preh }} \simeq \frac{2 \pi \Delta N_{\text {osc }}}{M_{\phi}} \sim 10^{-2} \mathrm{GeV}^{-1},
$$

where $\Delta N_{\text {osc }}$ counts the number of oscillations, and $M_{\phi}$ is the mass of the fields $\phi$ and $N$ in the global minimum (and therefore the typical frequency of their oscillations):

$$
M_{\phi}=\kappa\langle N\rangle=O(1 \mathrm{TeV}) .
$$

The decay rates of the fields involved, $\tilde{N}_{R_{1,2}}, H_{i}$, and singlets $\phi$ and $N$, can be estimated as:

$$
\begin{aligned}
\Gamma_{\tilde{N}_{R_{1}}} & \simeq \frac{\left|\lambda_{L R}\right|_{11}^{2}}{8 \pi} M_{R_{1}} \approx O(0.01) \mathrm{GeV}, \\
\Gamma_{\tilde{N}_{R_{2}}} & \simeq \frac{\left|\lambda_{L R}\right|_{22}^{2}}{8 \pi} M_{R_{2}} \approx O\left(10^{4}\right) \mathrm{GeV}, \\
\Gamma_{H_{i}} & \simeq \frac{g^{2}}{8 \pi} M_{H} \approx O(10) \mathrm{GeV}, \\
\Gamma_{\phi} & \simeq \frac{\kappa^{2}}{8 \pi} M_{\phi} \approx O\left(10^{-17}\right) \mathrm{GeV},
\end{aligned}
$$

\footnotetext{
${ }^{3}$ Due to the smalleness of the couplings, non-thermal production of gravitinos [27] is not a problem [28].
} 
where $g$ is the electroweak coupling constant, and the mass of the Higgses are $M_{H_{i}} \simeq$ $O(100 \mathrm{GeV}-1 \mathrm{TeV})$. The sneutrinos $\tilde{N}_{R_{2}}$ will tend to decay immediately after they are produced, $\Gamma_{\tilde{N}_{R_{2}}}^{-1} \ll \Delta t_{\text {preh }}$, and its decay products quickly thermalize by scattering from each other [29], given that:

$$
\Delta n_{l} \sigma_{s c}>H
$$

where $\Delta n_{l}$ is the number density of the light degrees of freedom $\Delta n_{l} \simeq n_{\tilde{N}_{2}}, \sigma_{s c} \propto$ $M_{R_{2}}^{-2}$, and $H$ is the Hubble parameter of the order $O(M e V)$. No back reaction is expected from them, except that part of the vacuum energy is converted into radiation with a temperature $T_{0} \simeq 0.3 c_{2}^{1 / 4} V(0)^{1 / 4}$. The fields $H_{i}$ and $\tilde{N}_{R_{1}}$ do not decay before $\Delta t_{\text {preh }}$. Therefore, back reaction effects due not only to the singlets but also to the Higgses (and eventually $\tilde{N}_{R_{1}}$ ) will soon slow down and suppressed the rate of production of particles during preheating, in less than a Hubble time $H^{-1}$.

At this point we can consider that the Universe has been reheated up to a temperature $T_{0} \sim O\left(10^{8} \mathrm{GeV}\right)$, but with a non-negligible fraction of the energy still in the form of cold oscillations and singlets, Higgses and $\tilde{N}_{R_{1}}$. The right handed sneutrinos $\tilde{N}_{R_{1}}$ also decay out-of-equilibrium in a time $\delta t \simeq \Gamma_{\tilde{N}_{R_{1}}}^{-1} \ll H^{-1}$, transferring its energy to the thermal bath. On the other hand, the decay rate of the Higgses will now be suppressed by the factor $M_{H} / T_{0}$, rendering it quite inefficient. Therefore, after preheating becomes inefficient, back reaction and rescattering effects take place, and both $\tilde{N}_{R_{2}}$ and $\tilde{N}_{R_{1}}$ decay, we are left with the vacuum energy distributed among the singlets, Higgses, and radiation.

Preheating can become very inefficient, but will not neccessarily stop as long as $\phi$ and $N$ continue to oscillate (that is, there is some energy density left in these fields), and production of Higgses from the singlets might continue in a very narrow resonance regime [23], at the same time than standard reheating. On the other hand, we do not expect this effect to be enough to further induced the production of the much heavier right handed sneutrinos. The preheating/reheating era will end when the singlets finally decay, at a time $t \simeq \Gamma_{\phi}^{-1}$.

If the masses of the singlets are smaller than the lightest Higgs mass, the fields $\phi$ and $N$ decay predominantly into axions, with the branching ratio into others particles being much smaller. The axions behave as relativistic particles, but they do not thermalize [30], that is, their interaction rate always remains smaller than the Hubble expansion parameter. The axion interaction rate $\Gamma_{A I}$ is given by:

$$
\Gamma_{A I}=\left\langle\sigma_{a}|v|\right\rangle n_{R}
$$

where $n_{R}$ is the radiation number density $n_{R} \simeq T^{3} / \pi^{2}$, and $\sigma_{a}$ is the axion cross section for scattering off the thermalize radiation. On dimensional grounds, the cross section can be written as $\sigma_{a}=\alpha_{a} / f_{a}^{2}, f_{a}$ being the axion decay constant $10^{12}-$ $10^{13} \mathrm{GeV}$.

\footnotetext{
${ }^{4}$ The coupling $\alpha_{a}$ is either due to tree-level interactions, and therefore further suppressed by a factor $T^{-2}$ when the temperature is larger than the typical mass scale of the particle exchanged, or due to loops effects, which are suppressed by a factor $1 /\left(8 \pi^{2}\right)^{2}$. Therefore, until the temperature drops near $O(1 \mathrm{GeV})$ we have $\alpha_{a} \sim 10^{-4}$.
} 
Let us define $t_{0}$ as the initial time after the initial burst of radiation produced by the sneutrinos decay. At this time, the Universe is at a temperature $T_{0} \sim O\left(10^{8} \mathrm{GeV}\right)$ but still $H \sim V(0)^{1 / 2} / \sqrt{3} M_{P}$. The ratio $\Gamma_{A I} / H$ is then:

$$
\left.\frac{\Gamma_{A I}}{H}\right|_{t_{0}} \simeq \frac{\alpha_{a}}{f_{a}^{2}} \frac{T_{0}^{3}}{\pi^{2}} \frac{\sqrt{3} M_{P}}{V(0)^{1 / 2}} \simeq 10 \alpha_{a} c^{3 / 4}\left(\frac{10^{12} G e V}{f_{a}}\right)^{2}<1 .
$$

After the Universe becomes radiation dominated at a temperature $T_{R D}$ we will have instead:

$$
\left.\frac{\Gamma_{A I}}{H}\right|_{t_{0}} \simeq \frac{\alpha_{a}}{f_{a}^{2}} \frac{1}{\pi^{2}}\left(\frac{90}{\pi^{2} g_{T}}\right)^{1 / 2} T M_{P}<10^{-5} \alpha_{a}\left(\frac{10^{12} G e V}{f_{a}}\right)^{2} T_{R D}<1,
$$

where we have used $H \simeq\left(\pi^{2} g_{T} / 90\right) T^{2} / M_{P}$, and $g_{T}$ is the effective number of relativistic degrees of freedom at $T$. The last inequality in Eq. (26) follows because the factor $\alpha_{a}$ becomes $O(1)$ only when $T \sim O(1 \mathrm{GeV})$. If radiation is being produced while the Universe is matter dominated, the number density and Hubble parameter evolve as $n_{R} \propto a^{-1 / 2}$ and $H \propto a^{-3 / 2}$, with $a$ the scale factor, so the ratio $\Gamma_{A I} / H$ increases in time. In a radiation dominated Universe they will both scale as $a^{-1 / 2}$. In either case, Eqs. (25) and (26) ensure that the axions never come into equilibrium. This means that we do not expect radiation to be produced from the singlets (inflaton), contrary to most models of inflation. Any extra radiation will come eventually from the Higgses decay.

Let us now briefly summarise the above discussion. We have argued that righthanded sneutrinos are produced during the initial period of preheating, but they decay rapidly into leptons and Higgses. We are now interested in the evolution of the Universe from this time until the time of reheating $t_{R H}$, defined as the time at which the singlets completely decay?. In this interval there is an interplay between the energy density of the oscillating inflaton fields $\rho_{\phi}$, the number density of Higgses $n_{H}$, the energy of the axion fields $\rho_{\text {axion }}$, and the energy density in radiation $\rho_{R}$. We shall model this as follows. $\rho_{\phi}$ will be steadily reduced due to continual production of axions (through the standard perturbative rate $\Gamma_{\phi a}$ ) and Higgses (through inefficient preheating). The axions behave as relativistic matter, but they stay out of equilibrium. Higgses are created and annihilate into radiation with a thermal-averaged crossed section $\left\langle\sigma_{H}|v|\right\rangle$ and decay rate $\left\langle\Gamma_{H}\right\rangle$. Therefore, the radiation density $\rho_{R}$ receive contributions from the Higgses but not from the singlets. On the other hand, among the decay products of the Higgses we will find also neutralinos, the lightest of them being a candidate for cold dark matter. The standard calculation of their relic abundance 31] depends mostly on their freeze-out temperature, the temperature at which they decouple from the plasma, and it is usually assumed that this happens while the Universe is radiation dominated. That is the case when the reheating temperature is much larger than the typical mass scale of the particle, that is, reheating ended much before they freeze-out. However, as shown in Ref. [32], the situation

\footnotetext{
${ }^{5}$ In general, the reheating time will coincide with the time at which the Universe becomes radiation dominated $t_{R D}$. However in the present context this may not be the case, see below.
} 
changes in an scenario with a low reheating temperature, such that freeze-out takes place when the Universe is still matter dominated. In fact it is shown that, for a B-ino like lightest neutralino, the cosmological constraints on the B-ino mass and/or the right handed slepton mass are relaxed and even disappear once a reheating temperature below the B-ino mass is allowed. In our type of scenario, moreover, the Higgses will be kept for a while out-of-equilibrium, due to preheating, and therefore neutralinos will be produced also out-of-equilibrium, initially most likely in a matter dominated universe. If they do not re-enter equilibrium before reheating is complete, their relic abundance may be different than that obtained in other scenarios.

Under the above assumptions, the evolution of the energy densities (singlets, axions and radiation) and number densities (Higgses and neutralinos) during reheating can be described by a simple set of equations [10, 11, 32]:

$$
\begin{aligned}
\dot{\rho}_{\phi}= & -3 H \rho_{\phi}-\Gamma_{\phi a} \rho_{\phi}-\Gamma_{p r e h} \rho_{\phi}, \\
\dot{\rho}_{\text {axion }}= & -4 H \rho_{\text {axion }}+\Gamma_{\phi a} \rho_{\phi}, \\
\dot{n}_{H}= & -3 H n_{H}-\left(1-B_{\chi}\right)\left\langle\Gamma_{H}\right\rangle\left(n_{H}-n_{H}^{e q}\right)-B_{\chi}\left\langle\Gamma_{H}\right\rangle\left(n_{H}-\frac{n_{\chi}^{2}}{n_{\chi}^{e q^{2}}} n_{H}^{e q}\right) \\
& -\left\langle\sigma_{H}|v|\right\rangle\left(n_{H}^{2}-n_{H}^{e q^{2}}\right)+\Gamma_{\text {preh }} \frac{\rho_{\phi}}{M_{H}}, \\
\dot{n}_{\chi}= & -3 H n_{\chi}+B_{\chi}\left\langle\Gamma_{H}\right\rangle\left(n_{H}-\frac{n_{\chi}^{2}}{n_{\chi}^{e q^{2}}} n_{H}^{e q}\right)-\left\langle\sigma_{\chi}|v|\right\rangle\left(n_{\chi}^{2}-n_{\chi}^{e q^{2}}\right), \\
\dot{\rho}_{R}= & -4 H \rho_{R}+\left(1-B_{\chi}\right)\left\langle\Gamma_{H}\right\rangle\left\langle E_{H}\right\rangle\left(n_{H}-n_{H}^{e q}\right) \\
& +2\left\langle\sigma_{H}|v|\right\rangle\left\langle E_{H}\right\rangle\left(n_{H}^{2}-n_{H}^{e q^{2}}\right)+2\left\langle\sigma_{\chi}|v|\right\rangle\left\langle E_{\chi}\right\rangle\left(n_{\chi}^{2}-n_{\chi}^{e q^{2}}\right),
\end{aligned}
$$

where $\Gamma_{\text {preh }}$ models the rate of production of Higgses through "inefficient" preheating, $\left\langle E_{H}\right\rangle$ and $\left\langle E_{\chi}\right\rangle$ are the average energy per Higgs and neutralino respectively, with

$$
\left\langle E_{i}\right\rangle \simeq \sqrt{m_{i}^{2}+T^{2}}, i=H, \chi
$$

and in the numerical calculations we have set the masses $m_{H}=m_{\phi}=10 m_{\chi}=1 \mathrm{TeV}$, and

$$
\begin{gathered}
\left\langle\Gamma_{H}\right\rangle \simeq \alpha_{H} m_{H}\left(1-\exp \left(-m_{H} / T\right)\right), \\
\left\langle\sigma_{i}|v|\right\rangle \simeq \frac{\alpha_{i}^{2}}{T^{2}}\left(1-\exp \left(-T^{2} / m_{i}^{2}\right)\right),
\end{gathered}
$$

with $\alpha_{H}=\alpha_{\chi}=10^{-3}$. The preheating period would set the initial conditions to solve these equations, and at $t_{0}=0$ we will take:

$$
\rho_{\phi} \simeq \rho_{R} \simeq\left\langle E_{H}\right\rangle n_{H} \simeq \frac{V(0)}{3}
$$

When reheating ends at $t=t_{R H}$, the temperature of the Universe will be $T_{R H} \propto$ $\sqrt{\Gamma_{\phi} M_{P}}$, where $M_{P}$ is the reduced Planck mass and $\Gamma_{\phi}=\Gamma_{\phi a}+\Gamma_{p r e h}$ the total decay rate of the singlets. 
To start with, the Higgses will soon approach equilibrium due to scattering process, with $\left\langle\sigma_{H}|v|\right\rangle n_{H}^{e q}>H\left(t_{0}\right)$. If $\Gamma_{\text {preh }} \ll \Gamma_{\phi}$, no appreciable amount of Higgses/radiation is further produced and the Higgses will later decay in equilibrium. This means that $\rho_{R}$ will be redshifted as $a^{-4}$, faster than the axions which are produced in singlets decay, $\rho_{\text {axion }} \propto a^{-3 / 2}$, and when reheating ends we will have an axion-dominated Universe. Given that the entropy would be conserved, and that $\rho_{\text {axion }}\left(t_{R H}\right) \simeq \rho_{\phi}\left(t_{R H}\right)$, one can estimated the final ratio of the energy density of axions to radiation as:

$$
\frac{\rho_{\text {axion }}\left(T_{R H}\right)}{\rho_{R}\left(T_{R H}\right)} \simeq \frac{\rho_{\phi}\left(t_{0}\right)}{\rho_{R}\left(t_{0}\right)}\left(\frac{T_{0}}{T_{R H}}\right) \approx 10^{8}
$$

The relativistic axions will behave as an extra generation of neutrinos at the time of nucleosynthesis, with the number of extra generations constraint by $\delta N_{\nu} \leq 1.8$ 222. This translates into a bound for the energy density of axions relative to that in radiation:

$$
\left.\frac{7}{8} \frac{\rho_{\text {axion }}}{\rho_{R}}\right|_{\text {nucl. }} \leq 1.8 .
$$

Comparing this bound with Eq. (36), it is clear that we need the Higgses out of equilibrium and start decaying into radiation at some time $t_{1}$ much before reheating is complete. This will be achieved taking $\Gamma_{\text {preh }}>\Gamma_{\phi a}$. This also means that allowing the standard perturbative decay of the singlets into Higgses does not solve the problem of axion dominance, since one expects the respective branching ratios into axions and Higgses to be at most of the same order. We need some extra effect apart from "perturbative" decay. However, if we take $\Gamma_{\text {preh }}$ as a constant parameter, and larger than $\Gamma_{\phi a}$, we are forcing the singlets to decay completely through preheating, which in a realistic scenario is unlikely to happen. It is more reasonable to consider this parameter as a decreasing function of time: as the energy density of the oscillating singlets decreases, the rate of production through preheating will also diminish. We will consider a simple ansatz where $\Gamma_{\text {preh }}$ follows an exponential law,

$$
\Gamma_{\text {preh }}(t)=\Gamma_{\text {preh }}\left(t_{0}\right) e^{-b t},
$$

with $b$ a constant such that $b<\Gamma_{\text {preh }}\left(t_{0}\right)^{-1}$. We can imagine this like $\Gamma_{\text {preh }}(t)$ being switched on for a while, until the energy density $\rho_{\phi}$ diminishes enough to make parametric resonance completely negligible at $t>b^{-1}$, when $\Gamma_{\text {preh }}(t)$ is switched off.

The situation now is as follows: the Higgses start decaying at a time $t_{1}<t_{R H}$, which can be estimated as:

$$
t_{1}^{-1} \sim H\left(t_{1}\right) \sim \frac{\rho_{\phi}\left(t_{1}\right)}{n_{H}\left(t_{1}\right)} \frac{\Gamma_{p r e h}\left(t_{0}\right)}{M_{H}} \sim \frac{T_{0}}{M_{H}} \Gamma_{p r e h}\left(t_{0}\right)
$$

and given that until this time the entropy is constant, and $T \propto a^{-1}$, we have:

$$
T_{1} \approx\left(.1 \frac{M_{P}}{M_{H}}\right)^{2 / 3} T_{0}^{1 / 3} \Gamma_{p r e h}^{2 / 3} \sim\left(10^{20} \Gamma_{\text {preh }}\right)^{2 / 3} \mathrm{GeV} .
$$

Higgs/radiation production stops at $t_{2} \simeq \Gamma_{\text {preh }}\left(t_{0}\right)^{-1}$. Inmediately after the Higgses decay. Therefore, entropy is only released between $T_{1}$ and $T_{2}$, and in that interval the 


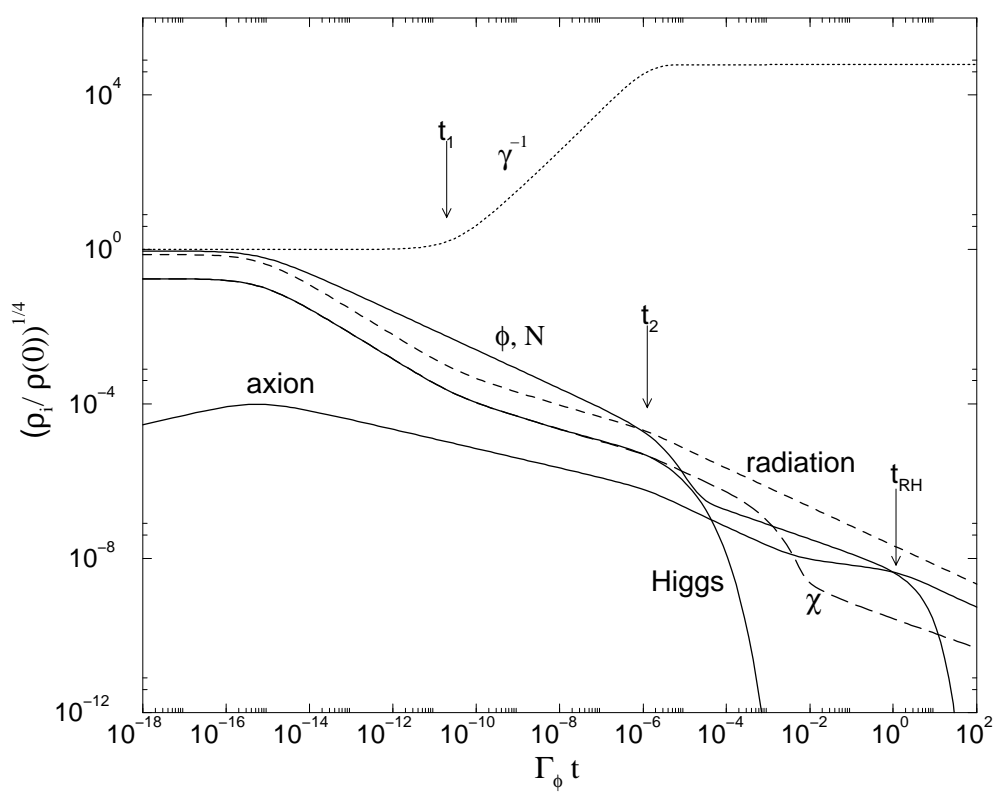

Figure 1: Evolution of the energy densities of the singlets $(\phi, N)$, axions, Higgses, radiation (dashed line) and neutralinos (long-dashed line), when $\Gamma_{p r e h}\left(t_{0}\right)=10^{-12} \mathrm{GeV}$, and $b=10^{5} \Gamma_{\phi}$. We have taken $B_{\chi}=0.5$. We have also included the factor $\gamma^{-1}=S_{f} / S_{i}$ (dotted line).

ratio $\rho_{\text {axion }} / \rho_{R}$ is frozen. After $t_{2}$ we are left still with the singlets producing more axions, so the ratio of axions to radiation again increases until $t_{R H}$ when is frozen again.

This can be seen in Fig. 1, where we have plotted the evolution of the energy densities for the case $\Gamma_{\text {preh }}\left(t_{0}\right)=10^{-12}>\Gamma_{\phi}$ and $b=10^{5} \Gamma_{\phi}$ as an example. The time scale is given in units of $\Gamma_{\phi}$. The singlets start decaying through $\Gamma_{\text {preh }}$ at $t \simeq t_{1}$, but still we have assumed that they initially dominated the energy density of the Universe. The Higgses initially are in equilibrium, but due to the contribution from $\Gamma_{\text {preh }}$ they start to decay into radiation and neutralinos. At this point the ratio $S_{f} / S_{i}$ starts to increase as can be seen in the figure. Entropy production stops at $t_{2}$, when the singlets energy density is partially depleted due to $\Gamma_{\text {preh }}$. The Universe becomes radiation dominated at a temperature $T_{2} \simeq T_{R D} \simeq 10^{3} \mathrm{GeV}$; this is larger than the lower reheating temperature we would expect only from $\Gamma_{\phi a}$. Soon after, inefficient preheating is swichted off and the Higgses decay. Until $\Gamma_{\phi} t \simeq O(1)$ we still produced more axions from the remaining singlets. The final ratio of the axions to radiation is $\rho_{\text {axion }} / \rho_{R} \sim 10^{-3}$, which is consistent with nucleosynthesis. The neutralinos follow the same evolution than the Higgses as far as both are relativistic. Once the Higgses decay, they go into equilibrium and when they become non-relativistic the ratio $n_{\chi} / s$ freezes out. At $T_{R H}$ we have $\left.\left(n_{\chi} / s\right)\right|_{R H} \simeq 4 \times 10^{-11}$, which would imply a relic abundance of neutralinos of order 1 today; they would dominate the dark matter in the Universe. We notice that in this example the neutralinos enter into equilibrium and freeze out in a radiation dominated Universe, so the calculation of their relic abundance would not differ from the standard one, and the usual bounds would apply. 
In the above example, the Universe becomes radiation dominated at a temperature $T_{2}<T_{1}$. However, from Eq. (40) we see that had we taken $\Gamma_{\text {preh }}\left(t_{0}\right) \geq 10^{-8}$, then $T_{1} \sim T_{0}$, and the Universe would be radiation dominated indeed during the whole period of what we call "reheating", that to say, until the singlets completely disappear.

With this in mind, we now turn to the calculation of the entropy dilution factor $\gamma$ :

$$
\gamma^{-1}=\left(\frac{S\left(T_{2}\right)}{S\left(T_{1}\right)}\right)=\left(\frac{T_{2}}{T_{1}}\right)^{3}\left(\frac{a\left(t_{2}\right)}{a\left(t_{1}\right)}\right)^{3},
$$

which will depend on the initial value of $\Gamma_{\text {preh }}\left(t_{0}\right)$, but not on its time dependence. If $\Gamma_{\text {preh }}\left(t_{0}\right) \leq 10^{-8}$, then the value of $T_{2}$ is given by the condition $\rho_{\phi}\left(t_{2}\right) \simeq \rho_{R}\left(t_{2}\right)$, and we obtain:

$$
\gamma^{-1} \approx 0.4\left(\frac{T_{2}}{T_{1}}\right)^{3} \frac{\rho_{\phi}\left(t_{1}\right)}{\rho_{R}\left(t_{2}\right)} \approx \frac{T_{0}}{\sqrt{\Gamma_{\text {preh }\left(t_{0}\right)} M_{P}}} \leq 10^{8} .
$$

The smaller the value of $\Gamma_{\text {preh }}\left(t_{0}\right)$, the larger the dilution becomes. On the other hand, for large values of $\Gamma_{\text {preh }}\left(t_{0}\right)$, entropy production takes place when the Universe is already radiation dominated, the radiation energy density scales as $\propto a^{-1}$, and the time $t_{2}$ is given by the condition $\rho_{\phi}\left(t_{2}\right) \approx \rho_{H}\left(t_{2}\right)$ instead. In this case, $\gamma$ tends to a constant value, given by:

$$
\gamma^{-1}=\left(\frac{a\left(t_{2}\right)}{a\left(t_{0}\right)}\right)^{3} \simeq\left(\frac{\rho_{\phi}\left(t_{0}\right)}{\rho_{H}\left(t_{0}\right)}\right)^{3 / 2} \approx 10^{4} \times\left(\frac{\rho_{\phi}\left(t_{0}\right)}{\rho_{R}\left(t_{0}\right)}\right)^{3 / 2} .
$$

Due to entropy production, the final ratio of axions to radiation given in Eq. (36) is also diluted, such that:

$$
\frac{\rho_{\text {axion }}\left(T_{R H}\right)}{\rho_{R}\left(T_{R H}\right)} \simeq \gamma \frac{\rho_{\phi}\left(t_{0}\right)}{\rho_{R}\left(t_{0}\right)}\left(\frac{T_{0}}{T_{R H}}\right) \times \exp \left(-F\left[\frac{\Gamma_{\text {preh }}\left(t_{0}\right)}{b}\right]\right) .
$$

The last factor in the above equation is due to the partial depletion in the energy density of the singlets around the time $t \simeq \Gamma_{\text {preh }}\left(t_{0}\right)^{-1}$, with:

$$
F[x]=x(1-\exp (1 / x)) .
$$

To summarize, in order to avoid axion dominance at the end of the reheating period, we have allowed the singlets to decay into Higgses through inefficient preheating, which will decay into radiation. The effect on the final ratio of axions to radiation is twofold: on one hand it reduces the ratio because part of the singlets have been converted into Higgses instead of axions; on the other hand, the final ratio gets also diluted by a factor $\gamma$ due to entropy production. However, the same factor $\gamma$ will dilute the initial lepton assymmetry produced in the s-neutrinos decays, which was our main concern. The lower dilution factor is obtained when what we have called "reheating" starts directly with a radiation dominated Universe instead of the 
usual matter-inflaton dominated Universe. Based on simplest assumptions, we have obtained an upper bound on $\gamma$,

$$
\gamma \sim 10^{-4} \times \frac{\rho_{R}\left(t_{0}\right)}{\rho_{\phi}\left(t_{0}\right)},
$$

which means that the baryon asymmetry at the end of reheating will be at most:

$$
\frac{n_{B}}{s} \sim 10^{-12 \pm 2}
$$

a couple of orders of magnitude below the observational data. Given that we have only considered a kind of toy model to study reheating, and the uncertainties in it, we regard this result as quite promising. In particular, we remark again that the value of $\Gamma_{\text {preh }}$ is an unknown in the model, controlled by the physics of preheating. Moreover, we have taken $\rho_{R}\left(t_{0}\right) \simeq \rho_{\phi}\left(t_{0}\right)$, but it may happen that we could produce more radiation than expected before reheating starts. Finally, we have considered a particular model for neutrinos masses as a working example, such that the values of the Yukawa couplings generate an asymmetry of the order $\epsilon_{1} \sim 10^{-8}$. Other texture models with values of the Yukawas consistent with the experimental data on neutrinos, and similar hierarchy among the right handed neutrinos, could give rise to a larger asymmetry such as $\epsilon_{1} \sim 10^{-6}$ [20], which would give then the correct order of magnitude for the baryon asymmetry.

In any case, the less dilution we can have, the better, and this translates into the Universe becoming radiation dominated as soon as possible. In general, this means that neutralino freeze out will take place in a radiation dominated Universe, and the standard bound on their relic abundance will apply.

Finally, we mention that we could also avoid axion dominance allowing the singlets to decay into other light degrees of freedom apart from axions. However, if the singlets decay into radiation this will imply a too large dilution factor, with [10]

$$
\gamma \sim s_{0} \frac{\sqrt{\Gamma_{\phi} M_{P}}}{\rho_{\phi}(0)} \sim 10^{-8},
$$

where the entropy density is $s_{0} \sim T_{0}^{3}$, and the energy density in singlets $\rho_{\phi}$ are taken as the initial values at $t_{0}$.

\section{Conclusion}

In this paper we have discussed a realistic supersymmetric model of inflation [14 which couples the inflaton to the Higgs, and when enlarged to include right-handed neutrinos allows leptogenesis as the mechanism to generate the observed baryon asymmetry of the Universe. From the particle physics point of view, the model is an extension of the Next-to-Minimal Supersymmetric Model and solves the $\mu$ problem via an intermediate scale which generates the vev for the singlets. The interaction between 
the singlets and matter fields are dictated by an approximate $U(1)$ Peccei-Quinn symmetry, providing also a solution for the strong $\mathrm{CP}$ problem, and the axions as a candidate for dark matter. As a hybrid inflationary model, it has a quite low scale for inflation $\left(O\left(10^{8} \mathrm{GeV}\right)\right.$, and its predicts an spectral index $n=1$ consistent with the recent Boomerang and Maxima-1 data [33], and in principle a very low reheating temperature of the order of a few GeV, barring the possibility of both GUT and electroweak baryogenesis. Therefore we have extended the model to include right-handed neutrinos, and have appealed to preheating to produce the lightest right-handed sneutrino so that baryogenesis may proceed via leptogenesis.

Extending the model to include heavy right handed neutrinos is one of the preferred solutions not only for the sake of leptogenesis, but in order to generate a light neutrino mass spectrum through the see-saw mechanism, given the strong evidence from experiments in support of such light masses and mixings. In this paper we have used as an example a realistic model of all quark and lepton masses and mixing angles [16] based on single right handed neutrino dominance [17]. We have chosen this particular example because it predicts not only a hierarchical spectrum for the light neutrinos but also for the heavy right handed neutrinos/sneutrino and in particular involves a relatively light right handed state which is available for preheating, although we emphasise that this spectrum came out of an analysis of neutrino masses and mixing angles which was not performed with leptogenesis in mind. The right handed neutrinos and sneutrinos are too heavy to be produced thermally at any stage in our inflationary model. However, we have argued that at least the lightest right handed sneutrino can be produced through parametric resonance during preheating, due to its couplings to the Higgs doublets. Its CP-violating decay gives rise to a lepton asymmetry, later converted by sphalerons into baryon number. Note that the right handed sneutrinos do not couple directly to the oscillating inflaton fields, but rather indirectly via the Higgs doublets. Thus Higgs scalars are also expected to be produced during reheating, and since these decay into radiation and neutralinos we may estimate the amount of neutralinos and entropy that is produced during preheating as shown in Figure 1. Note that relativistic axions are also produced during reheating but these are red-shifted away, although later on non-relativistic axions are additionally produced by the usual misalignment mechanism and these will contribute to cold dark matter.

Because the heavy sneutrinos decay long before reheating is completed, the lepton asymmetry will be subsequently diluted by the entropy produced in the decays of singlets and Higgses, before the time of nucleosynthesis. In order to avoid too much dilution, we first required the singlets to decay only into axions, which do not thermalize and do not contribute to the radiation energy. The radiation energy density has its origin in the out-of-equilibrium decay of Higgses and sneutrinos, which have been previously produced during preheating. If no more radiation is produced, by the time the singlets completely decay the Universe becomes axion-dominated, violating by many orders of the magnitude the bound on the number of extra relativistic neutrino-like species at nucleosynthesis. We argue then than "inefficient" preheating of the Higgses is required in order to allow their out-of-equilibrium decay and some 
extra production of radiation. Due to the long life-time of the singlets, it is possible that, while they are oscillating, they could "preheat" other fields, at a rate similar or even larger than the perturbative decay rate. To illustrate this point we have presented a simplified analyses of the "reheating" period, parametrising "inefficient" preheating by a rate $\Gamma_{p r e h}$, in order to compare it with the perturbative decay rate $\Gamma_{\phi}$, and it is the result of this simplified analysis which is presented in Figure [1]. The main qualitative conclusion is that in order to avoid axion dominance we would require $\Gamma_{\text {preh }}>\Gamma_{\phi}$. Therefore, the main parameter controlling the analyses is the ratio of these decay rates (the perturbative one and through inefficient preheating), which will also depend to some extent on the ratio of the Higgs and singlets masses. Note that the lighter the Higgses are, the easier they will be produced. However, this may modify the period of preheating following the end of inflation. The more radiation which is produced the less the dilution we will have later (Eq. (46).

To summarise, we have presented a semi-quantitative scenario for leptogenesis in the context of a realistic supersymmetric low scale hybrid inflationary model. A novelty of the model is that during the reheating period, the dilution of the leptonbaryon asymmetry is not due to entropy produced in the inflaton decays but due to Higgs decay. This in turn is controlled by how many Higgses we are able to "preheat" from the singlets before reheating is finally completed, and it will help to avoid an axion-dominated Universe at the end of reheating. In a model with a hierarchy in the masses of the heavy right handed neutrinos, the combination of the small asymmetry $\epsilon$ with some later dilution could give rise to the correct order of magnitude for the final value of $n_{B} / s$. Note that in a realistic supersymmetric model such as this the value of the baryon number is related to the question of the nature and abundance of cold dark matter, and that these questions are in turn related to the questions of the $\mu$ problem, the strong $\mathrm{CP}$ problem, and neutrino masses.

\section{Acknowledgements}

S.K. acknowledges the support of a PPARC Senior Fellowship. We thank Vicente Di Clemente for reading the manuscript, Toni Riotto for comments, and Gordy Kane for numerous comments and encouragement.

\section{References}

[1] M. Fukugita and T. Yanagida, Pjys. Lett. B174 (1986) 45.

[2] M. A. Luty, Phys. Rev. D45 (1992) 455.

[3] H. Murayama, H. Suzuki and T. Yanagida, Phys. Rev. Lett. 70 (1993) 1912.

[4] B. A. Campbell, S. Davidson and K. A. Olive, Nucl Phys. B399 (1993) 111.

[5] H. Murayama and T. Yanagida, Phys. Lett. B322 (1994) 349; T. Moroi and H. Murayama, hep-ph/9908223. 
[6] G. t'Hooft, Phys. Rev. Lett. 37 (1976) 8; V. A. Kuzmin, V. A. Rubakov and M. E. Shaposhnikov, Phys. Lett. B155 (1985) 36; J. Ambjrn, T. Askgaard, H. Porter and M. E. Shaposhnikov, Nucl. Phys. B353 (1991) 346.

[7] Super-Kamiokande collaboration, Phys. Lett. B436 (1998) 33.; The Soudan 2 collaboration, Phys. Let.. B449 (1999) 137; The MACRO collaboration, Phys. Lett. B434 (1998) 451.

[8] W. Buchmüller and T. Yanagida, Phys. Lett. B302 (1993) 240; W. Buchmüller and M. Plümacher, Phys. lett. B389 (1996) 73; M. Flanz, E. A. Paschos, U. Sarkar and J. Weiss, Phys. Lett. B389 (1996) 693; A. Pilaftsis, Phys. Rev. D56 (1997) 5431; W. Buchmüller and M. Plümacher, Phys. Lett. B431 (1998) 354; W. Buchmüller and T. Yanagida, Phys. Lett. B445 (1999) 399; J. Ellis, S. Lola and D. V. Nanopoulos, hep-ph/9902364; M. S. Berger and B. Brahmachari, hep-ph/9801271; R. Barbieri, P. Creminelli, A. Strumia and N. Tetradis, hep$\mathrm{ph} / 9911315$.

[9] For a review, see S. Sarkar, Rep. Prog. Phys. 59 (1996) 1493; for the latest analysis, see E. Holtmann, M. Kawasaki, K. Kohri and T. Moroi, Phys. Rev. D60 (1999) 023506.

[10] E. W. Kolb and M. S. Turner, The Early Universe, (Addison-Wesley, Menlo Park, Ca. 1990).

[11] D. J. Chung, E. W. Kolb and A. Riotto, Phys. Rev. D60 (1999) 063504.

[12] T. Asaka, K. Hamaguchi, M. Kawasaki and T. Yanagida, hep-ph/9907559.

[13] G. F. Giudice, M. Peloso, A. Riotto and I. Tkachev, JHEP 8 (1999) 14.

[14] M. Bastero-Gil and S. F. King, Phys. Lett. B 423 (1998) 27.

[15] M. Bastero-Gil and S.F. King, Nucl. Phys. B549 (1999) 391.

[16] S.F.King and M.Oliveira, hep-ph/0009287.

[17] S.F.King, Phys. Lett. B439 (1998) 350; S.F.King, Nucl. Phys. B562 (1999) 57; S.F.King, Nucl. Phys. B576 (2000) 85; S.F.King and N.N.Singh, Nucl. Phys. B591 (2000) 3 .

[18] L. Covi, E. Roulet and F. Vissani, Phys. Lett. B384 (1996) 169; L. Covi, E. Roulet and F. Vissani, Phys. Lett. B424 (1998) 101.

[19] For review, see A. Pilaftsis, hep-ph/9812256; W. Buchmüller and M. Plümacher, hep-ph/9904310.

[20] See for example M. S. Berger and B. Brahmachari, in [8].

[21] S. Yu. Khlebnikov and M. E. Shaposhnikov, Nucl. Phys. B308 (1988) 885; J. S. Harvey and M. S. Turner, Phys. Rev. D42 (1990) 3344. 
[22] See, for example, K. A. Olive, G. Steigman and T. P. Walker, astro-ph/9905320.

[23] J. H. Traschen, R. H. Branderberger, Phys. Rev. D 42 (1990) 2491; L. A. Kofman, A. D. Linde and A. A. Starobinsky, Phys. Rev. Lett. 73 (1994) 3195; Y. Shtanov, J. Traschen and R. Branderberger, Phys. Rev. D 51 (1995) 5438.

[24] Lev Kofman, Andrei Linde, Alexei Starobinsky, Phys. Rev. D 56 (1997) 3258. S. Yu. Khlebnikov, I. I. Tkachev, Phys. Rev. Lett. 77 (1996) 219; T. Prokopec and T. G. Roos, Phys. Rev. D 55 (1997) 3768; S. Yu. Khlebnikov, I. I. Tkachev, Phys. Rev. Lett. 79 (1997) 1607; Juan García-Bellido, Andrei Linde, Phys.Rev. D 57 (1998) 6075;

[25] J. Baacke, K. Heitmann and C. Patzold, Phys. Rev. D58 (1998) 125013; G. Felder, L. Kofman and A. Linde, hep-ph/9812289; P. B. Greene and L. Kofman, Phys. Lett B448 (1999); A. L. Maroto and A. Mazumdar, Phys. Rev. D59. (1999) 083510.

[26] M. Bastero-Gil, S. F. King and J. Sanderson, Phys. Rev. D60 (1999) 103517.

[27] A. Maroto and A. Mazumdar, Phys. Rev. Lett. 84 (2000) 1655; R. Kallosh, L. Kofman, A. Linde and A. V. Proeyen, Phys. Rev. D61 (2000) 103503; hepth/0006354; G. F. Giudice, I. Tkachev and A. Riotto, JHEP 08 (1999) 009; D. H. Lyth, Phys. Lett. B469 (1999) 69; 476 (2000) 356; A. L. Maroto and J. R. Pelaez, Phys. Rev. D62 (2000) 023518;

[28] M. Bastero-Gil and A. Mazumdar, hep-ph/0002004.

[29] J. Ellis, K. Enqvist, D. V. Nanopoulos and K. A. Olive, Phys. Lett. B191 (1987) 343.

[30] K. Choi, E. J. Chun and J. E. Kim, Phys. Lett. B403 (1997) 209.

[31] R. J. Scherrer and M. S. Turner, Phys. Rev. D33 (1986) 1585; K. A. Olive and M. Srednicki, Phys. Lett. B230 (1989) 78; R. Arnowitt and P. Nath, Phys. Rev. Lett. 70 (1993) 3696; M. Drees and M. M. Nojiri, Phys. Rev. D47 (1993) 376; G. L. Kane, C. Kolda, L. Roszkowski and J. D. Wells, Phys. Rev. D49 (1994) 6173; J. Ellis, R. Falk and K. A. Olive, Phys. Lett. B444 (1998) 367; J. Ellis, T. Falk, K. A. Olive and M. Srednicki, Astropart. Phys. 13 (2000) 181.

[32] G. F. Giudice, E. W. Kolb and A. Riotto, hep-ph/0005123.

[33] P. de Bernardis et al. (Boomerang Coll.), Nature 404 (2000) 955; S. Hanany et al. (Maxima Coll.), astro-ph/0005123 